\title{
Postoperative changes in platelet adhesiveness
}

\author{
P. N. BENNETT \\ From the Department of Medicine, University of Aberdeen
}

SYNOPSIS Platelet adhesiveness was measured in patients undergoing operation. With the rotating bulb technique adhesiveness increased steadily to the sixth and ninth days following surgery. The glass bead filter technique demonstrated a marked increase in platelet adhesiveness on the day following operation.

The relationship of altered platelet behaviour to the thrombotic tendency following surgical operations has attracted interest in recent years. Payling Wright (1942) using a rotating bulb technique found that the increase in platelet stickiness was maximal on the tenth postoperative day. Platelet clumping activity was found to be increased postoperatively by Emmons and Mitchell (1965), and Hampton and Mitchell (1966) observed platelet electrokinetic response to be greatest on the day after operation followed by a progressive decline to normal levels.

The glass bead filter technique for measuring platelet adhesiveness introduced by Hellem (1960) is now widely used. In a preliminary study of the postoperative state with this technique the pattern of change in adhesiveness was found to be different from that previously reported with the rotating bulb technique (Payling Wright, 1942) though both methods rely on the adhesion of platelets to glass. A comparative study was therefore undertaken of the glass bead filter and rotating tube techniques in patients undergoing surgical operations.

\section{METHODS AND SUBJECTS}

Platelet adhesiveness by Hellem's method was measured by counting platelets in citrated venous blood issuing from a motor-driven syringe before and after the interposition of a standard glass bead filter $(5 \mathrm{~g}$. ballotini no. 8 beads) with a contact time of 24.5 seconds. The difference between the two platelet counts represents the number of platelets retained in the filter and this number is expressed as a percentage of the pre-filter or venous count. Filters were made in batches of 20 and all studies for any one patient were performed on filters from the same batch.

Platelet adhesiveness with the modification of Payling Wright's original method now used (McDonald and Edgill, 1958) was measured by rotating citrated venous blood at $3 \frac{1}{2}$ r.p.m. in a glass bulb for 20 minutes. The

Received for publication 9 March 1967. difference in the platelet count before and after rotation represents the number of platelets lost by adhesion to the glass surface and this number is expressed as a percentage of the pre-rotation or venous count.

No correction of haematocrit was made before testing blood samples.

Platelet counts were performed on citrated blood diluted 1:20 with $1 \%$ ammonium oxalate using phase contrast microscopy and counting approximately 750 platelets in each of two counting chambers for each count.

Haematocrit was measured in a Hawksley microhaematocrit centrifuge.

The subjects were male and female patients in two general surgical wards who underwent cholecystectomy (4), vagotomy and gastro-enterostomy (5), nephrectomy (1), prostatectomy (1), and vesicolithotomy (1). Two patients received 1 pint of blood at operation; none of the remainder had blood transfusion. Platelet adhesiveness was measured on blood from the same sample with the glass bead filter technique at 15 minutes and with the rotating bulb technique commencing rotation at 17 minutes after venepuncture on the day before and on the first, third, sixth, and ninth days following operation.

\section{RESULTS}

The accompanying table shows that the mean venous count did not alter during the first three postoperative days following which a thrombocytosis occurred. The percentage of adhesive platelets as measured by the glass bead filter technique increased in all subjects on the first postoperative day $(P<0.001)$ and this level was maintained on the third postoperative day after which the percentage of adhesive platelets 6 declined. The absolute number of adhesive platelets with this method increased markedly on the first postoperative day and afterwards increased more gradually till the sixth day after operation.

With the rotating bulb technique the percentage and absolute number of adhesive platelets did not $\stackrel{\unrhd}{\unrhd}$ alter significantly on the first postoperative day but $\underset{\sigma}{\sigma}$ 
TABLE

MEAN CHANGES WITH S.E. IN PLATELET COUNT AND ADHESIVENESS IN 10 PATIENTS BEFORE AND AFTER OPERATION

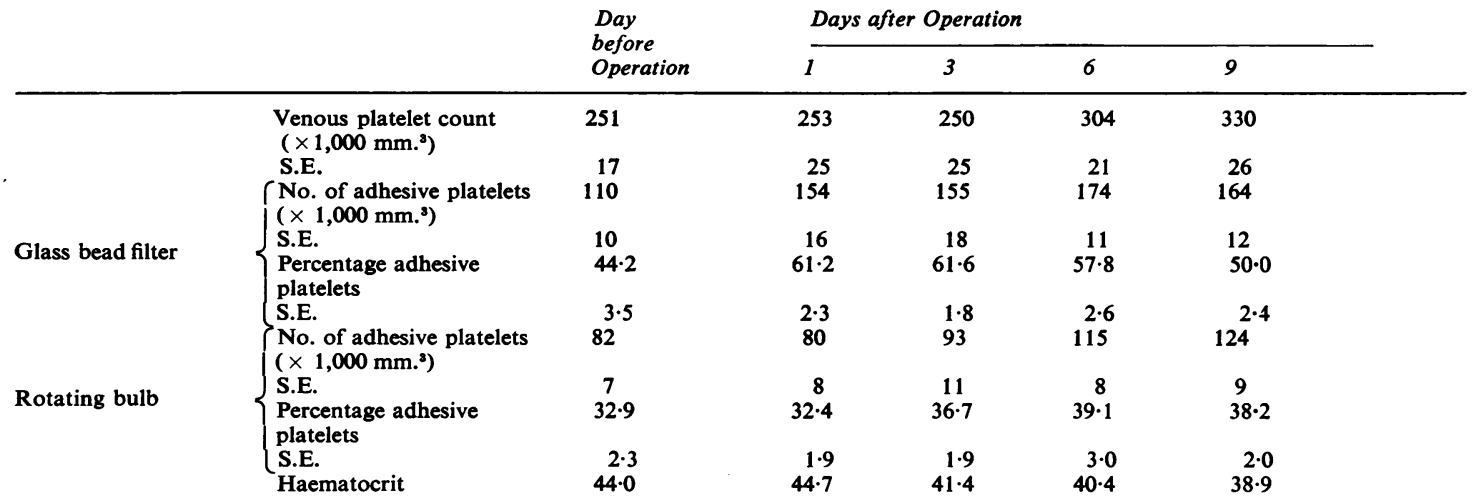

thereafter rose to maximal levels on the sixth and ninth days following operation.

Haematocrit did not change significantly on the day following operation but thereafter declined slowly.

\section{DISCUSSION}

Using a rotating bulb technique Payling Wright (1942) found increasing platelet adhesiveness following surgery until the tenth postoperative day. This finding is confirmed in the present study. Platelet adhesiveness as measured by the glass bead filter technique, however, showed a considerable increase on the first three post-operative days following which the percentage of adhesive platelets fell although the absolute number of adhesive platelets rose. Changing haematocrit and a tendency for measured adhesiveness to increase slightly with filter age (Bennett, Bennett, Fullerton, and Ogston 1966) make the degree of change in adhesiveness after the first postoperative day difficult to evaluate precisely. It is clear, however, that the two techniques are responding in a different manner to the same situation, and the explanation could either be that the glass bead filter method is more sensitive or that the two techniques are measuring different aspects of platelet activity. The higher level of adhesiveness constantly recorded by the glass bead filter methods is a feature of the chosen experimental conditions and need not itself explain the difference in response.

It seems possible that the activity of a platelet population may be governed in part by its absolute number of adhesive platelets and in part by its pro- portion of adhesive platelets. In either case the observations with the glass bead filter technique suggest that following operation there is an immediate increase in platelet activity.

Venous thrombosis is known to occur throughout the postoperative period, but relatively less frequently in the days immediately following surgery. There is, however, experimental evidence of a delay between the stimulus to thrombosis and the phlebitic reaction (Marin and Stefanini, 1960). Furthermore, Bygdeman, Eliasson, and Johnson (1966) have shown that an increase in A.D.P.- induced platelet adhesiveness precedes the appearance of signs of thrombosis by some days. If venous thrombosis in the second postoperative week is the result of earlier events, the demonstration of enhanced platelet adhesiveness early in the postoperative period might assume greater significance.

I wish to acknowledge the advice and criticism of Professor H. W. Fullerton in the preparation of this paper and to thank Professor G. Smith and Mr. N. J. Logie for allowing me to study patients under their care.

The work was assisted by a grant from the Tobacco Research Council on the recommendation of the British Heart Foundation.

\section{REFERENCES}

Bennett, N. B., Bennett, P. N., Fullerton, H. W., Ogston, C. M., and Ogston, D. (1966) Lancet, 2, 881.

Bygdeman, S., Eliasson, R., and Johnson, S. R. (1966). Ibid., 1, 1301. Emmons, P. R., and Mitchell, J. R. A. (1965). Ibid.. 1, 71.

Hampton, J. R., and Mitchell, J. R. A. (1966). Brit. med. J., 1, 1078. Hellem, A. J. (1960). Scand. J. clin. Lab. Invest., 12, suppl. 51.

McDonald, L., and Edgill, M. (1958). Lancet, 1. 996.

Marin, H. M., and Stefanini, M. (1960) Surg. Gynec. Obstet., 110, 263. Wright, H. P. (1942). J. Path. Bact., 54, 461. 